International Journal of Geometric Methods in Modern Physics

(C) World Scientific Publishing Company

\title{
Boundary Effects in Bosonic and Fermionic Field Theories
}

\author{
M. Asorey \\ Departamento de Física Teórica, Facultad de Ciencias, Universidad de Zaragoza \\ E-50009 Zaragoza, Spain \\ asorey@unizar.es \\ D. García-Alvarez \\ Departamento de Análisis Económico \\ Facultad de Economía y Empresa. \\ Universidad de Zaragoza. E-50005 Zaragoza. Spain \\ dga@unizar.es \\ J. M. Muñoz-Castañeda \\ Institut für Theoretische Physik, Universität Leipzig, \\ Brüderstr. 16, D-04103 Leipzig, Germany \\ jose.munoz-castaneda@uni-leipzig.de \\ Received (Day Month Year) \\ Revised (Day Month Year)
}

\begin{abstract}
The dynamics of quantum field theories on bounded domains requires the introduction of boundary conditions on the quantum fields. We address the problem from a very general perspective by using charge conservation as a fundamental principle for scalar and fermionic quantum field theories. Unitarity arises as a consequence of the choice of charge preserving boundary conditions. This provides a powerful framework for the analysis of global geometrical and topological properties of the space of physical boundary conditions. Boundary conditions which allow the existence of edge states can only arise in theories with a mass gap which is also a physical requirement for topological insulators.
\end{abstract}

Keywords: boundary conditions; charge conservation; unitarity, edge states.

\section{Introduction}

Since the early days of the quantum theory boundary effects arise in quantum physics. In Young's double slit experiments, which provide many key ingredients of the foundations of the quantum theory, boundary effects play a crucial role. Boundary effects also appear as relevant ingredients in the Aharonov-Bohm effect, which points out the quantum observability of phase factors of electromagnetic fields.

More recently, a plethora of new quantum effects induced by the presence of boundaries boosted a new era of quantum technologies. Some of the most remark- 
able new phenomena include the Casimir effect, the presence of plasmons and other surface effects in metals and dielectrics, the appearance of edge currents in the Hall effect, the opening of a gap in small graphene samples and new edge effects in topological insulators.

From a more basic viewpoint boundary effects also appear in fundamental physics: black hole horizons effects, Hawking radiation, topological defects, topology change and holographic effects in the AdS/CFT correspondence.

The increasing relevance of boundary effects is demanding a comprehensive theory of boundary conditions. In spite of the fact that quite a lot of work has been devoted to establish the foundations of the quantum theory, a comprehensive theory of boundary conditions for quantum theories is still missing. A first attempt to fill the gap was initiated by Asorey-Ibort-Marmo in Ref. [1] and was further developed in [2. In this paper we will try to emphasize some new aspects of this approach in a relativistic context and illustrate the emerging general theory by means of examples.

In quantum mechanics the fundamental principle of the theory of boundary conditions is the preservation of probability. Indeed, unitarity imposes severe constraints on the boundary behavior of quantum states in systems confined to bounded domains [1. However, in relativistic field theories, the fundamental principle is charge conservation, which together with causality imposes further conditions [2]. The space of boundary conditions compatible with both constraints has interesting global geometric properties. The dependence of many interesting physical phenomena, like the Casimir effect [3], topology change [415|6] or renormalization group flows [7, on the boundary conditions can be analyzed from this global perspective.

\section{Boundary effects in Quantum Mechanics}

To illustrate the large variety of boundary conditions which are compatible with the foundations of quantum mechanics let us consider a free particle of unit mass confined in an one dimensional interval $[0, L]$. Even in classical mechanics the dynamics of a free particle is not completely defined by the Hamiltonian

$$
H=\frac{p^{2}}{2} \text {. }
$$

Once the particle reach the boundaries at $x=0$ or $x=L$ one needs to specify how the particle bounces back into the interval. There are several possibilities, which depend on the physical properties of the boundaries: complete reflection, sticky reflection or even a complete stop of the particle motion at the boundary (see [8]). In quantum mechanics the situation is similar. The naive quantization rule gives rise to a Hamiltonian

$$
H=-\frac{1}{2} \frac{d^{2}}{d x^{2}}
$$

which is not selfadjoint operator in $L^{2}([0, L])$ and, thus, does not univocally determines the quantum dynamics. 
Even if $H$ is symmetric for smooth functions of compact support there are many other functions on $L^{2}([0, L])$ where $H$ is not symmetric. For instance for two arbitrary smooth functions on $[0, L]$ we have

$$
\left(\psi_{1}, H \psi_{2}\right)=-\frac{1}{2} \int_{0}^{L} \psi_{1}^{*} \psi_{2}^{\prime \prime} d x
$$

By integrating by parts one obtains

$$
\left(\psi_{1}, H \psi_{2}\right)-\left(\psi_{2}, H \psi_{1}\right)=-\frac{1}{2} \int_{0}^{L} \frac{d}{d x}\left[\psi_{1}^{*} \psi_{2}^{\prime}-\psi_{1}^{\prime *} \psi_{2}\right]=\Sigma\left(\psi_{1}, \psi_{2}\right) .
$$

The obstruction to the symmetry of $H$ arises from the boundary term

$$
\Sigma\left(\psi_{1}, \psi_{2}\right)=\psi_{1}^{*} \psi_{2}^{\prime}(L)-\psi_{1}^{\prime *} \psi_{2}(L)-\psi_{1}^{*} \psi_{2}^{\prime}(0)-\psi_{1}^{\prime *} \psi_{2}(0) .
$$

This boundary term can only vanish on a dense subset of functions on $L^{2}([0, L]$ if there is an $2 \times 2$ unitary matrix

$$
U=\left(\begin{array}{ll}
u_{11} & u_{12} \\
u_{11} & u_{22}
\end{array}\right)
$$

such that the boundary values of the functions in the domain of the Hamiltonian satisfy the following boundary conditions [19]

$$
\left(\begin{array}{c}
\varphi(0)-i \dot{\varphi}(0) \\
\varphi(L)-i \dot{\varphi}(L)
\end{array}\right)=\left(\begin{array}{ll}
u_{11} & u_{12} \\
u_{21} & u_{22}
\end{array}\right)\left(\begin{array}{c}
\varphi(0)+i \dot{\varphi}(0) \\
\varphi(L)+i \dot{\varphi}(L)
\end{array}\right)
$$

where $\dot{\varphi}(0)=-\psi^{\prime}(0)$ and $\dot{\varphi}(L)=\psi^{\prime}(L)$. In other terms, the definition of quantum dynamics has many different implementations parametrized by the different selfadjoint extension of $H$, which are in one to one correspondence with the unitary matrices of $U(2)[19]$.

Some specially interesting examples correspond to the case when the matrix $U$ is diagonal or anti-diagonal. In the first case we have

$$
U_{\alpha}=\left(\begin{array}{cc}
e^{-i \alpha_{1}} & 0 \\
0 & e^{-i \alpha_{2}}
\end{array}\right)
$$

which corresponds to the Robin boundary conditions

$$
\begin{aligned}
-\sin \frac{\alpha_{1}}{2} \varphi(0)+\cos \frac{\alpha_{1}}{2} \dot{\varphi}(0) & =0 \\
-\sin \frac{\alpha_{2}}{2} \varphi(L)+\cos \frac{\alpha_{2}}{2} \dot{\varphi}(L) & =0
\end{aligned}
$$

which includes Newmann $\dot{\varphi}(0)=\dot{\varphi}(L)=0$ and Dirichlet $\varphi(0)=\varphi(L)=0$ boundary conditions. In the anti-diagonal case

$$
U_{\epsilon}=\left(\begin{array}{cc}
0 & e^{-i \epsilon} \\
e^{i \epsilon} & 0
\end{array}\right)
$$

we have pseudo-periodic boundary conditions $\varphi(L)=e^{i \epsilon} \varphi(0)$ with a probability flux propagating from one boundary to the other. The $U_{0}=\sigma_{1}$ matrix with $\epsilon=0$ 
corresponds to periodic boundary conditions $\varphi(0)=\varphi(L)$ and that with $\epsilon=\pi$ to anti-periodic boundary conditions $\varphi(0)=-\varphi(L)$.

Another interesting non-diagonal case is described by the unitary matrix

$$
U_{g}=\frac{1}{1-i g}\left(\begin{array}{cc}
i g & 1 \\
1 & i g
\end{array}\right)
$$

which corresponds to a delta like potential on a point of a circle 9610]11. This

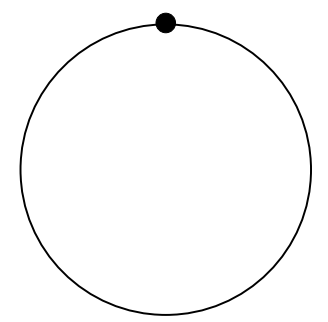

Fig. 1. The dynamics of a superconducting quantum device (SQUID) can be described by the boundary conditions introduced by the matrix $U_{g}$ of equation 3

boundary condition describes the effective dynamics of circular superconducting quantum device (SQUID) with a Josephson junction (see Figure 1). In this case the non-diagonal form of the boundary condition is accounting for the tunneling effects from one side of the interval to the other, which in this particular case corresponds to a delta like potential in the circle 910. All other non-diagonal boundary conditions correspond to more general contact interactions combined with magnetic fluxes crossing the circle.

The generalization for several intervals is straightforward 411. In the case of two intervals $[-2 L,-L] \cup[L, 2 L]$ the boundary conditions are given by

$$
\left(\begin{array}{c}
\varphi(-2)-i \dot{\varphi}(-2) \\
\varphi(-L)-i \dot{\varphi}(-L) \\
\varphi(L)-i \dot{\varphi}(L) \\
\varphi(2 L)-i \dot{\varphi}(2 L)
\end{array}\right)=\left(\begin{array}{llll}
u_{11} & u_{12} & u_{13} & u_{14} \\
u_{21} & u_{22} & u_{23} & u_{24} \\
u_{31} & u_{32} & u_{33} & u_{34} \\
u_{41} & u_{42} & u_{43} & u_{44}
\end{array}\right)\left(\begin{array}{c}
\varphi(-2)+i \dot{\varphi}(-2) \\
\varphi(-L)+i \dot{\varphi}(-L) \\
\varphi(L)+i \dot{\varphi}(L) \\
\varphi(2 L)+i \dot{\varphi}(2 L)
\end{array}\right),
$$

in terms of a four dimensional unitary matrix $U \in U(4)$, where $\dot{\varphi}(-2 L)=$ $-\psi^{\prime}(-2 L), \dot{\varphi}(-L)=\psi^{\prime}(-L) \dot{\varphi}(L)=-\psi^{\prime}(L)$ and $\dot{\varphi}(2 L)=\psi^{\prime}(2 L)$. The nondiagonal elements of the boundary conditions describe boundary conditions where the global conservation of the probability flux is obtained by cancellation of the non-trivial magnetic fluxes between the four different edges of the intervals. For instance, the unitary matrix

$$
U_{1}=\left(\begin{array}{llll}
0 & 0 & 0 & 1 \\
0 & 0 & 1 & 0 \\
0 & 1 & 0 & 0 \\
1 & 0 & 0 & 0
\end{array}\right)
$$


corresponds to periodic boundary conditions between the edges $-L$ and $L$ and $2 L$ and $-2 L$ which describe the quantum dynamics on circle of length 2 , whereas the unitary matrix

$$
U_{2}=\left(\begin{array}{llll}
0 & 1 & 0 & 0 \\
1 & 0 & 0 & 0 \\
0 & 0 & 0 & 1 \\
0 & 0 & 1 & 0
\end{array}\right)
$$

corresponds to periodic boundary conditions between the edges $-2 L$ and $-L$, and $L$ and $2 L$ which describe the quantum dynamics on two independent circles of unit length. Thus, the transition from a double connected topology to a single connected one described in Figure 2 can be carried out in a smooth way by changing the boundary conditions along the one-parameter family $U_{s}=U_{1}^{s} U_{2}^{1-s}, s \in[0,1]$ of unitary matrices of $U(4)$, interpolating between the matrices $U_{2}$ and $U_{1}$. In this way a topology fluctuation phenomena can be described in very a simple manner in terms of boundary conditions [15]6].
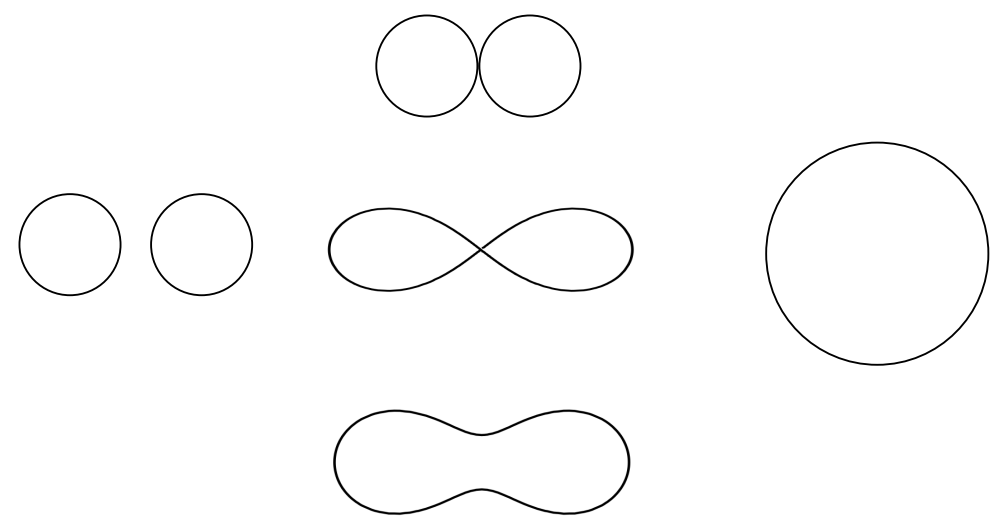

Fig. 2. Topology change induced by a continuous change of boundary conditions $U_{s}=U_{1}^{s} U_{2}^{1-s}, s \in$ $[0,1]$. The transition from a two circle topology $U_{2}$ to one circle topology $U_{1}$ is made through a contact point topology $U_{\infty}$.

The absolute principle of strict conservation of the quantum probability in a bounded domain $\Omega \subset \mathbb{R}$ is not realistic, because most of the physical boundaries are

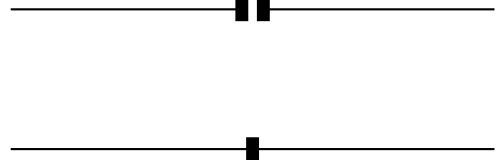

Fig. 3. The effect of a singular boundary between two complementary domains can be described by boundary conditions on the inner/outer interface boundary between the two domains. 
not completely insulating. The most general boundary conditions should account for more realistic boundaries, where a net probability flux can cross the boundary and the absolute law of probability conservation only holds in the whole space. This can be achieved by doubling the boundary $\partial \Omega$ and considering two systems: an inner system in $\Omega$ and an outer system in $\mathbb{R} \backslash \Omega$, both sharing a common boundary $\partial \Omega$.

Let us illustrate the method with a simple example. Let us consider, for simplicity the splitting of the real line into two unbounded semi-intervals $(-\infty,-L]$ and $[L, \infty)$ (see Figure 3). It is easy to check that the only boundary conditions that cancel probability flux in the whole system are again given by

$$
\left(\begin{array}{c}
\varphi(-L)-i \dot{\varphi}(-L) \\
\varphi(L)-i \dot{\varphi}(L)
\end{array}\right)=\left(\begin{array}{cc}
u_{11} & u_{12} \\
u_{21} & u_{22}
\end{array}\right)\left(\begin{array}{c}
\varphi(-L)+i \dot{\varphi}(-L) \\
\varphi(L)+i \dot{\varphi}(L)
\end{array}\right)
$$

in terms of a $2 \times 2$ unitary matrix $U \in U(2)$. The boundary condition (3) given by the matrix $U_{g}$ in this case corresponds to a $-g \delta(x)$ potential sitting in the origin (as $L \rightarrow 0$ ) of the real line $\mathbb{R}$, i.e.

$$
\begin{gathered}
\varphi(L)=\varphi(-L) \\
\varphi^{\prime}(L)-\varphi^{\prime}(-L)=-2 g \varphi(L) .
\end{gathered}
$$

In the case of an attractive potential $-g \delta(x)$ with $g>0$, there is a negative energy bound state of the form $\psi(x)=\sqrt{g} e^{-g|x|}$ 12[13|14, which corresponds to a physical state highly localized at the impurity, i.e. an edge state. The same state also appears as a negative mode for the Robin boundary conditions with a diagonal unitary matrix of type 22 with $\tan \frac{\alpha_{1}}{2}=\tan \frac{\alpha_{1}}{2}=-g$ and $\pi<\alpha_{1}=\alpha_{2}<2 \pi$. This is general feature than can happen for boundary conditions whose unitary matrices have an eigenvalue $e^{i \alpha}$ with $\pi<\alpha<2 \pi[12$, which provides natural candidates of suitable boundary conditions for topological insulators with edge states [15]16].

\section{Boundary conditions and charge conservation}

We have shown that unitarity is the fundamental quantum principle of the theory of boundary conditions in non-relativistic quantum mechanics. However, boundary conditions also appears in many other classical and quantum physical systems governed by partial differential equations as necessary conditions to uniquely determine the dynamical evolution of the physical system when is confined on a bounded domain.

In the theory of boundary conditions in quantum mechanics there is another equivalent principle, which can be easily generalized and allows the extension of the formalism beyond the framework of non-relativistic quantum mechanics. Indeed, we have seen that unitarity is related to probability conservation, but this is also is related to charge conservation, which ultimately is a consequence the existence of a $U(1)$ internal symmetry. Indeed, the conservation of the $U(1)$ symmetry can be used as the fundamental principle in the theory of physical boundary conditions. 
In the presence of a $U(1)$ symmetry Noether's theorem implies the existence of a conserved current

$$
\partial^{\mu} j_{\mu}=0
$$

Thus, the dynamical variation of the charge $\rho=j_{0}$ in a given subdomain $\Omega$ can be expressed in terms of the net flux of the current $\vec{j}$ across its boundary boundary $\partial \Omega$,i.e.

$$
\frac{d}{d t} \int_{\Omega} \rho d v=\int_{\partial \Omega} \vec{j} \cdot d \vec{\sigma}
$$

A good example is non-relativistic quantum mechanics, where the Schrödinger equation $i \partial_{t} \Psi=-\frac{1}{2} \nabla^{2} \psi+V(x) \psi$ preserves the $U(1)$ symmetry

$$
\psi^{\prime}(x)=e^{i \alpha} \psi(x) .
$$

The corresponding conserved current

$$
\vec{j}=\frac{i}{2}\left[\psi^{*} \vec{\nabla} \psi-\left(\vec{\nabla} \psi^{*}\right) \psi\right]
$$

encodes the probability conservation law.

This example also illustrate how two quantum symmetries can be related beyond simple compatibility. In this case unitarity of time evolution, or which is equivalent, time translation invariance, is equivalent to the existence of $U(1)$ charge symmetry. In other terms, charge conservation is strongly related to the self-adjointness of the Hamiltonian operator $H$ governing the time evolution. Indeed, the probability conservation law implies that

$$
\partial_{t}\langle\Psi(t) \mid \psi(t)\rangle=\left\langle\partial_{t} \Psi(t) \mid \psi(t)\right\rangle+\left\langle\Psi(t) \mid \partial_{t} \psi(t)\right\rangle=i\left\langle\Psi(t) \mid\left(H^{\dagger}-H\right) \psi(t)\right\rangle=0,
$$

i.e. the self-adjointness of the Hamiltonian operator $H, H^{\dagger}=H$. Conversely, selfadjointness of the Hamiltonian operator implies the unitary of time evolution and, thus, probability conservation. In this case both principles are equivalent. To better understand the crucial role played by semi-bounded quadratic forms in the theory of boundary conditions see Ref. [17].

\section{Boundary conditions in Scalar Field Theories}

In a complex scalar field theory which preserves the electric charge, i.e. the $U(1)$ gauge symmetry

$$
\phi^{\prime}(x)=e^{i \alpha} \phi(x),
$$

gives rise to the conserved current

$$
j_{\mu}=\frac{i}{2}\left[\phi^{*} \partial_{\mu} \psi-\left(\partial_{\mu} \phi^{*}\right) \phi\right] .
$$

Notice that in this case the charge density

$$
\rho=j_{0}=\frac{i}{2}\left[\phi^{*} \partial_{t} \psi-\left(\partial_{t} \phi^{*}\right) \phi\right]
$$


is not definite positive. Thus, the connection with unitary time evolution is not so evident. First of all, there is not a positive Hilbert product to associate with an unitary evolution. However, charge conservation can still be a good fundamental principle to define consistent boundary conditions when the system is confined in a bounded spatial domain $\Omega \subset \mathbb{R}^{3}$. Physically consistent boundary conditions must enforce the vanishing of the charge flux across the boundary $\partial \Omega$ of the system. In fact, since the spatial current is the same as in non-relativistic quantum mechanics, the theory of boundary conditions is also the same. Indeed, the boundary term accounting for the charge flux across the boundary is

$$
\Sigma(\phi)=\int_{\partial \Omega} \vec{j} \cdot d \vec{\sigma}=\frac{i}{2} \int_{\partial \Omega}\left[\phi^{*} \vec{\nabla} \phi-\left(\vec{\nabla} \phi^{*}\right) \phi\right] d \vec{\sigma} .
$$

If we now consider a linear combination $\phi=\phi_{1}+\alpha \phi_{2}$ of two independent fields $\phi_{1}, \phi_{2}$, such that $\Sigma\left(\phi_{1}\right)=\Sigma\left(\phi_{2}\right)=0$, we obtain

$$
\Sigma(\phi)=\frac{i \alpha}{2} \int_{\partial \Omega}\left[\phi_{1}^{*} \vec{\nabla} \phi_{2}-\left(\vec{\nabla} \phi_{1}^{*}\right) \phi_{2}\right] d \vec{\sigma}+\frac{i \alpha^{*}}{2} \int_{\partial \Omega}\left[\phi_{2}^{*} \vec{\nabla} \phi_{1}-\left(\vec{\nabla} \phi_{2}^{*}\right) \phi_{1}\right] d \vec{\sigma}
$$

which implies that

$$
\int_{\partial \Omega}\left[\phi_{1}^{*} \vec{\nabla} \phi_{2}-\left(\vec{\nabla} \phi_{1}^{*}\right) \phi_{2}\right] d \vec{\sigma}=\int_{\partial \Omega}\left[\phi_{2}^{*} \vec{\nabla} \phi_{1}-\left(\vec{\nabla} \phi_{2}^{*}\right) \phi_{1}\right] d \vec{\sigma}=0,
$$

and if denote by $\partial_{n}=\vec{\nabla} \cdot \vec{n}$ the normal derivative at the boundary $\partial \Omega$,

$$
\int_{\partial \Omega}\left[\phi_{1}^{*} \partial_{n} \phi_{2}-\left(\partial_{n} \phi_{1}^{*}\right) \phi_{2}\right] d \sigma=0
$$

Thus, the most general boundary condition without singular zero-modes, which preserves the charge density conservation law, is given by

$$
\left(1-i \partial_{n}\right) \phi=U\left(1+i \partial_{n}\right) \phi
$$

in terms of a unitary matrix $U$ defined on the boundary Hilbert space $L^{2}(\Omega)$ space. Notice that the above boundary conditions guarantee that the spacial Laplacian operator $-\Delta_{U}$ is selfadjoint with respect to the standard product $(\cdot, \cdot)$ of $L^{2}(\Omega)$ [1. In fact, all these selfadjoint extensions of $-\Delta$ can be defined by (7) in terms of unitary matrices of the Hilbert space $L^{2}(\Omega)[18 \mid 2$.

Let us consider a boundary condition such that $-\Delta_{U}$ is a positive operator. Then, we can define a pseudo-Hilbert product in the subspace $\mathcal{D}\left(\sqrt{-\Delta_{U}}\right) \oplus$ $\mathcal{D}\left(\sqrt{-\Delta_{U}}\right)$ of Hilbert space $\mathcal{H}=\mathcal{H}_{+} \oplus \mathcal{H}_{-}$, with $\mathcal{H}_{+}=\mathcal{H}_{-}=L^{2}(\Omega)$, given by

$$
(\phi, \phi)_{H}=\left(\phi_{+}, \sqrt{-\Delta_{U}} \phi_{+}\right)-\left(\phi_{-}, \sqrt{-\Delta_{U}} \phi_{-}\right)
$$

for any $\phi_{+}, \phi_{-}$in the domain $\mathcal{D}\left(\sqrt{-\Delta_{U}}\right)$ of $\sqrt{-\Delta_{U}}$ with $\phi=\phi_{+}+\phi_{-} \in \mathcal{H}$. Notice that the Hamiltonian of the associated free field theory $H= \pm \sqrt{-\Delta_{U}}$ is selfadjoint with respect to the $(\cdot, \cdot)_{H}$ product,

$$
(\phi, H \phi)_{H}=\left(\phi_{+}, H^{2} \phi_{+}\right)+\left(\phi_{-}, H^{2} \phi_{-}\right) .
$$


But not any selfadjoint extension of $H^{2}$ corresponds to one of $H$ with respect to the product $(\cdot, \cdot)_{H}$, only the positive ones satisfy this property. Finally, we remark that the pseudo hermitian product $(\cdot, \cdot)_{H}$ is nothing but the product associated to the conserved charge density (9).

An interesting remark is that the Hamiltonian of the free field theory is selfadjoint with respect to both products $(\cdot, \cdot)$ and $(\cdot, \cdot)_{H}$. There is, however, a significative difference between both cases. $H$ is not definite positive or negative with respect to the standard product $(\cdot, \cdot)$ of $\mathcal{H}_{+} \oplus \mathcal{H}_{-}$

$$
(\phi, H \phi)=\left(\phi_{+}, H \phi_{+}\right)+\left(\phi_{-}, H \phi_{-}\right)=\left(\phi_{+}, \sqrt{-\Delta_{U}} \phi_{+}\right)-\left(\phi_{-}, \sqrt{-\Delta_{U}} \phi_{-}\right),
$$

whereas with respect to the $H$-product it is selfadjoint and positive (8).

Finally, we remark that although we have emphasized the role of charge conservation in the definition of selfadjoint Hamiltonian for the free field theory, there are extra requirements which further constrain the range of physically consistent boundary conditions. In particular, as it was already mentioned, unitarity of the quantum field theory also requires that the operator $-\Delta_{U}$ has to be positive, to make possible the self-adjointness of the restriction of the Hamiltonian of the quantum field theory to the one-particle states $\sqrt{-\Delta_{U}}$ 2. This means that only boundary conditions defined by unitary matrices with eigenvalues $e^{i \alpha}$ with $0 \leq \alpha \leq \pi$ are physically consistent for any size of the physical space $\Omega[2]$.

\section{Boundary conditions in Fermionic Field Theories}

In a fermionic Dirac field theory which preserves the electric charge, the $U(1)$ gauge symmetry

$$
\psi^{\prime}(x)=e^{i \alpha} \psi(x),
$$

defines the conserved current is

$$
j_{\mu}=\bar{\psi} \gamma_{\mu} \psi
$$

Notice that in this case, unlike the bosonic case, the charge density is definite positive

$$
\rho=j_{0}=\bar{\psi} \gamma_{0} \psi=\psi^{\dagger} \psi
$$

Charge conservation becomes again a fundamental principle to define consistent boundary conditions. Physically consistent boundary conditions should imply the vanishing of the charge current flux

$$
\vec{j}=\bar{\psi} \vec{\gamma} \psi=\psi^{\dagger} \gamma_{0} \vec{\gamma} \psi
$$

across the boundary of the system $\partial \Omega$. The boundary term accounting for the charge flux across the boundary is

$$
\Sigma(\psi)=\int_{\partial \Omega} \vec{j} \cdot d \vec{\sigma}=\int_{\partial \Omega} \psi^{\dagger} \gamma_{0} \vec{\nabla} \psi d \vec{\sigma}=\int_{\partial \Omega} \psi^{\dagger} \gamma_{0} \vec{\gamma} \cdot \vec{n} \psi d \sigma .
$$


Again, as in the bosonic case, if we consider a linear combination $\psi=\psi_{1}+\alpha \psi_{2}$ of two independent fields $\psi_{1}, \psi_{2}$, such that $\Sigma\left(\psi_{1}\right)=\Sigma\left(\psi_{2}\right)=0$, we obtain

$$
\Sigma(\psi)=\alpha \int_{\partial \Omega} \psi_{1}^{\dagger} \gamma_{0} \vec{\gamma} \cdot \vec{n} \psi_{2} d \sigma+\alpha^{*} \int_{\partial \Omega} \psi_{2}^{\dagger} \gamma_{0} \vec{\gamma} \cdot \vec{n} \psi_{1} d \sigma
$$

which implies that the vanishing condition of the charge flux of $\psi$ is

$$
\int_{\partial \Omega} \psi_{1}^{\dagger} \gamma_{0} \vec{\gamma} \cdot \vec{n} \psi_{2} d \sigma=\int_{\partial \Omega} \psi_{2}^{\dagger} \gamma_{0} \vec{\gamma} \cdot \vec{n} \psi_{1} d \sigma=0
$$

The boundary term can be split as the difference of two positive chiral components

$$
\int_{\partial \Omega} \psi_{1}^{\dagger} \gamma_{0} \vec{\gamma} \cdot \vec{n} \psi_{2} d \sigma=\int_{\partial \Omega} \psi_{1}^{+\dagger} \psi_{2}^{+} d \sigma-\int_{\partial \Omega} \psi_{1}^{-\dagger} \psi_{2}^{-} d \sigma
$$

where $\psi^{ \pm}=\left(1 \pm \gamma_{0} \vec{\gamma} \cdot \vec{n}\right) \psi$.

Thus, the most general boundary condition preserving charge conservation is given by $\psi^{-}=U \gamma_{0} \psi^{+}$, i.e.

$$
\left(1-\gamma_{0} \vec{\gamma} \cdot \vec{n}\right) \psi=U \gamma_{0}\left(1+\gamma_{0} \vec{\gamma} \cdot \vec{n}\right) \psi,
$$

where $U$ is any unitary operator of the Hilbert space of boundary spinors, which anti-commute with $\gamma_{0} \vec{\gamma} \cdot \vec{n}$, i.e. $\left\{U, \gamma_{0} \vec{\gamma} \cdot \vec{n}\right\}=0$ [4].

The striking feature is that even if we select conservation of charge as the fundamental fundamental principle to fix the boundary conditions of the theory, we get as a bonus that these boundary conditions guarantee that the Hamiltonian of the free field theory $H=i \gamma^{0} \vec{\gamma} \cdot \vec{\nabla}-m \gamma^{0}$ is selfadjoint with respect to the product defined by the charge form

$$
\left(\psi_{1}, \psi_{2}\right)=\int_{\Omega} \psi_{1}^{\dagger} \psi_{2} d v
$$

i.e.

$$
\left(\psi_{1}, H \psi_{2}\right)=\left(H \psi_{1}, \psi_{2}\right) .
$$

This property together with time independence of the Dirac Hamiltonian $\left(\partial_{t} H=0\right)$ also implies time translation invariance of the free field theory

$$
\begin{aligned}
\partial_{t}(\psi, H \psi) & =\left(\partial_{t} \psi^{\dagger}, H \psi\right)+\left(\psi^{\dagger},\left(\partial_{t} H\right) \psi\right)+\left(\psi^{\dagger}, H \partial_{t} \psi\right) \\
& =i\left(H \psi^{\dagger}, H \psi\right)-i\left(\psi^{\dagger}, H^{2} \psi\right)=i\left(\psi^{\dagger},\left(H^{\dagger}-H\right) H \psi\right)=0 .
\end{aligned}
$$

By construction the Hilbert product $(\cdot, \cdot)$ is time translation invariant but the boundary conditions imply that this property is also preserved by the dynamics of the system, which guarantees the self-adjointness of the free field Hamiltonian, even if in this case it is not a positive operator.

In consequence, even if we give up the choice of unitarity as the fundamental principle to fix the boundary conditions of the system, we finally have a selfconsistent approach where charge conservation is the driving fundamental principle and unitarity is also recovered as a byproduct. 


\section{Acknowledgements}

Many of the results summarized in this paper were obtained in papers writen in collaboration with many other authors to whom we would like to thank: N. Acharya, A.P. Balachandran, J. Clemente-Gallardo, A. Ibort, G. Marmo, J.M. Pérez-Prado, A. Queiroz and S. Vaidya. M. A. work has been partially supported by the Spanish MICINN grants FPA2012-35453 and CPAN Consolider Project CDS2007-42 and DGA-FSE (grant 2014-E24/2).

\section{References}

[1] A. Asorey, A. Ibort and G. Marmo, Int. J. Mod. Phys. A 20 (2005) 1001

[2] M. Asorey and J. M. Muñoz-Castañeda, Nucl. Phys. B 874 (2013) 852;

[3] M. Asorey, D. García-Alvarez and J. M. Muñoz-Castañeda, J. Phys. A 39 (2006) 6127.

[4] A. P. Balachandran, G. Bimonte, G. Marmo and A. Simoni, Nucl. Phys. B446 (1995) 299.

[5] A.D. Shapere, F. Wilczek, Z. Xiong, Models of topology change, arXiv:1210.3545 (2012)

[6] M. Asorey et al, Quantum Physics and Fluctuating Topologies: Survey, arXiv:1211.6882 [hep-th] (2012).

[7] M. Asorey, D. García-Alvarez and J. M. Muñoz-Castañeda, J. Phys. A 40 (2007) 6767.

[8] M. Asorey, unpublished (1994)

[9] I. Tsutsui, T. Fülöp and T. Cheon Phys. Lett. A 264, (2000) 366374

[10] T. Fülöp and I. Tsutsui, J. Math. Phys. 42 (2001) 5687

[11] T. Cheon, T. Fülöp and I. Tsutsui, Ann. Phys. 294 (2001) 25

[12] M. Gadella, J. Negro and L.M. Nieto, Phys. Lett. A 373(2009) 13101313

[13] J. M. Munoz-Castaneda, J. Mateos Guilarte and A. Moreno Mosquera, Phys.Rev. D87 (2013) 105020

[14] J.M. Munoz-Castaneda, J. Mateos Guilarte, arXiv:1407.4212 preprint.

[15] H Zhang, CX Liu, XL Qi, X Dai, Z Fang and SC Zhang, Nature Phys. 5(2009) 438-442

[16] M. Asorey, A.P. Balachandran and J.M. Pérez-Pardo, JHEP 12 (2013) 073

[17] A. Ibort, F. LLedó and J.M. Pérez-Pardo, J. Funct. Anal. 268, 634-670 (2015)

[18] M. Asorey and J. M. Muñoz-Castañeda, Intern. J. Theor. Phys. 50 (2011) 2211. 